\title{
Dirac equation and the Melvin metric
}

\author{
L. C. N. Santos ${ }^{\mathrm{a}}$, C. C. Barros Jr. ${ }^{\mathrm{b}}$ \\ Depto de Física-CFM, Universidade Federal de Santa Catarina, CP. 476, Florianópolis, SC CEP 88.040-900, Brazil
}

Received: 2 February 2016 / Accepted: 29 September 2016 / Published online: 20 October 2016

(C) The Author(s) 2016. This article is published with open access at Springerlink.com

\begin{abstract}
A relativistic wave equation for spin 1/2 particles in the Melvin space-time, a space-time where the metric is determined by a magnetic field, is obtained. The energy levels for these particles are obtained as functions of the magnetic field and compared with the ones calculated with the Dirac equation in the flat Minkowski space-time. The numeric values for some magnetic fields of interest are shown. With these results, the effects of very intense magnetic fields on the energy levels, as intense as the ones expected to be produced in magnetars or in ultra-relativistic heavy-ion collisions, are investigated.
\end{abstract}

\section{Introduction}

In the past few years systems with extreme magnetic fields $\left(B>10^{15} \mathrm{G}\right)$ have been proposed to exist. In the magnetar analysis, for example, magnetic fields of the order of $10^{15} \mathrm{G}$ at the surface $[1,2]$ and $10^{17} \mathrm{G}$ at the center are expected to exist. In ultra-relativistic heavy-ion collisions, at $\sqrt{s}=200$ $\mathrm{GeV}$ (at RHIC), the magnetic field is expected to reach values as high as $10^{19} \mathrm{G}[3-5]$ and at LHC, at $\sqrt{s}=7 \mathrm{TeV}, 10^{20} \mathrm{G}$.

When studying particles inside these systems (electrons inside stars, and so on), the usual way is to solve the Dirac equation [6,7], for example, and find the energy levels, and in general, the Minkowski space-time is considered. A question that is quite intriguing is: if the magnetic field reach these values, may some effect of the structure of the space-time be observed? To address this question is the purpose of this work.

Including the magnetic field in the metric is not a trivial question but some solutions exist, as the Melvin metric [8-12], where a magnetic universe, with a magnetic field in the $z$ direction, is considered, or the Gutsunaev solution $[13,14]$, for a magnetic dipole. Of course it is always pos-

\footnotetext{
a e-mail: luis.santos@ufsc.br

b e-mail: barros.celso@ufsc.br
}

sible to study an arbitrary shape of the magnetic field and solve (at least numerically) the resulting Einstein equations, but with the objective of finding analytical results, and with this procedure, exploring this effect in a first approximation, we will consider the Melvin metric and find the wave equations for Dirac particles subject to a magnetic field inside this metric.

So, this paper will show the following contents: in Sect. 2 , a brief review of the formulation of the Dirac equation in curved spaces will be made. In Sect. 3 the wave equation in the Melvin metric will be worked out. The results and conclusions of this work will be presented in Sect. 4 .

\section{Dirac equation in curved spaces}

In this section a brief review of the wave equation for spin $1 / 2$ particles in curved spaces will be given. The basic formulation and the equations that will be needed in the next sections will be shown.

A fundamental characteristic of the Dirac equation is its invariance under Lorentz transformations, so, when studying Dirac particles in curved spaces, it is interesting to preserve this aspect. One way to do that is by using the tetrads $e_{\mu}^{(a)}$ that may be defined in order to satisfy the equation

$g_{\mu \nu}=e_{\mu}^{(a)} e_{\nu}^{(b)} \eta_{(a)(b)}$

where $\eta_{(a)(b)}$ is the Minkowski tensor, which represents a flat space-time, and $g_{\mu \nu}$ the metric tensor related to a space-time that possesses an arbitrary geometry [15-17].

Observing Eq. (1), we may note that the tetrads may be used in order to project vectors from the curved space-time in the flat space-time with the expression

$V_{\mu}=e_{\mu}^{(a)} V_{(a)}$

which relates the forms of a vector in different space-time geometries. 
As said before, the Dirac equation when written in the Minkowski geometry possesses Lorentz symmetry, and we want that the equivalent equation written in a curved spacetime possesses the same characteristic. In fact, if we study the behavior of the elements of the Dirac equation under transformations that preserve the desired symmetries, we may understand which are the quantities that need to be added or modified in the equation.

We must note that a spinor transforms according to

$\psi \rightarrow \rho(\Lambda) \psi$,

where $\rho(\Lambda)=1+\frac{1}{2} i \varepsilon^{(a)(b)} \Sigma_{(a)(b)}$, and $\Sigma_{(a)(b)}$ is the spinorial representation of the generators of the Lorentz transformation, written in terms of the $\gamma^{(c)}$ matrices, $\Sigma_{(a)(b)} \equiv$ $\frac{1}{4} i\left[\gamma_{(a)}, \gamma_{(b)}\right][18]$. The idea is to construct a covariant derivative $\nabla_{(a)} \psi$ that is locally Lorentz invariant, which means that we need to impose the transformation condition

$\nabla_{(a)} \psi \rightarrow \rho \Lambda_{(a)}^{(b)} \nabla_{(b)} \psi$

The usual way to obtain the form of the covariant derivative operator of the spinor is by assuming the combination

$\nabla_{(a)} \psi=e_{(a)}^{\mu}\left(\partial_{\mu}+\Omega_{\mu}\right) \psi$,

and considering the form of the operator $\Omega_{\mu}$ transformation

$\Omega_{\mu} \rightarrow \rho \Omega_{\mu} \rho^{-1}+\partial_{\mu} \rho \rho^{-1}$

If we choose the combination of terms

$\Omega_{\mu} \equiv \frac{1}{2} i \Gamma_{\mu}^{(a)(b)} \Sigma_{(a)(b)}=\frac{1}{2} i e_{\nu}^{(a)} \nabla_{\mu} e^{(b) v} \Sigma_{(a)(b)}$,

or in an equivalent form

$\Omega_{\mu} \equiv \frac{1}{2} i \Gamma_{(a) \mu(b)} \Sigma^{(a)(b)}$,

with the term $\Gamma_{(a) \mu(b)}$ defined as

$\Gamma_{(a) \mu(b)}=e_{(a) \nu}\left(\partial_{\mu} e_{(b)}^{\nu}+\Gamma_{\mu \lambda}^{\nu} e_{(b)}^{\lambda}\right)$,

where $\Gamma_{\mu \lambda}^{v}$ are the Christoffel symbols, Eq. (6) is satisfied. Consequently Eq. (5) satisfies the transformation condition required in (4) and then we get the final form of the covariant derivative operator

$\nabla_{(c)} \psi=e_{(c)}^{\mu}\left(\partial_{\mu}+\frac{1}{2} i e_{\nu}^{(a)} \nabla_{\mu} e^{(b) v} \Sigma_{(a)(b)}\right) \psi$.

We must remark that there are different definitions of covariant derivatives of tensors in the literature when studying the covariant Dirac equation, as for example in [19-21].
Considering the Dirac equation in a flat space-time,

$i \gamma^{(a)} \partial_{(a)} \psi-m \psi=0$,

and replacing the conventional derivative operator by the one obtained in (10) we obtain the desired wave equation for spin $1 / 2$ particles in a curved space-time

$i e_{(a)}^{\mu} \gamma^{(a)}\left(\partial_{\mu}+\Omega_{\mu}\right) \psi-m \psi=0$.

If the particle is submitted to an external electromagnetic field, we may introduce this effect by a minimal coupling

$i e_{(a)}^{\mu} \gamma^{(a)}\left(\partial_{\mu}+\Omega_{\mu}+i e A_{\mu}\right) \psi-m \psi=0$,

and the scalar action that leads to this equation is given by

$$
\begin{aligned}
S= & \int \mathrm{d}^{4} x \sqrt{-g} \bar{\psi}\left[i \gamma^{(c)} e_{(c)}^{\mu}\right. \\
& \left.\times\left(\partial_{\mu}+\frac{1}{2} i e_{\nu}^{(a)} \nabla_{\mu} e^{(b) v} \Sigma_{(a)(b)}+i e A_{\mu}\right)-m\right] \psi
\end{aligned}
$$

It is usual to define the term $\gamma^{\mu}=e_{(a)}^{\mu} \gamma^{(a)}$ as a Dirac matrix in a given curved space-time and it is easy to verify that it satisfies the Clifford algebra

$\gamma^{\mu} \gamma^{\nu}+\gamma^{\nu} \gamma^{\mu}=g^{\mu \nu}$.

\section{Wave equation in the Melvin metric}

If now we want to obtain the wave equations shown in the last section, in a space-time that has its structure determined by a magnetic field, a useful idea would be to consider the Melvin metric [8-12]. The Melvin metric is a solution of the Einstein-Maxwell equations of general relativity that represents a cylindrical magnetic universe. In his work [8,9], Melvin considered a static magnetic field where its lines lie in cylindrical surfaces perpendicular to the radial direction, with intensity $\sim B_{0}$ in the vicinity of the symmetry axis and falls as fast as $B_{0} / r^{4}$ far away from the axis. This solution has wide-ranging applications in the literature, as, for example in the study of Kerr black holes [22], or in cosmology, where the possibility of the interaction of the magnetic field with the expansion of the universe may be considered, as for example in [23,24].

The line element may be written in a cylindrically symmetric form (taking $c=G=\hbar=1$ ),

$\mathrm{d} s^{2}=\Lambda(r)^{2} \mathrm{~d} t^{2}-\Lambda(r)^{2} \mathrm{~d} r^{2}-\Lambda(r)^{-2} r^{2} \mathrm{~d} \phi^{2}-\Lambda(r)^{2} \mathrm{~d} z^{2}$, 
where $\Lambda(r)=1+\frac{1}{4} B_{0}^{2} r^{2}$ and $B_{0}$ is the magnetic field. This metric reflects the curvature of the space-time, determined by the existence of a magnetic field in the $z$ direction. For this reason, a metric with axial symmetry is taken into account.

In the limit of vanishing the magnetic field we have $\Lambda(r)=1$ and Eq. (16) becomes

$\mathrm{d} s^{2}=\mathrm{d} t^{2}-\mathrm{d} r^{2}-r^{2} \mathrm{~d} \phi^{2}-\mathrm{d} z^{2}$,

which is the flat Minkowski space-time written in cylindrical coordinates. So, we may define

$\eta_{(a)(b)}=\left(\begin{array}{cccc}1 & 0 & 0 & 0 \\ 0 & -1 & 0 & 0 \\ 0 & 0 & -1 & 0 \\ 0 & 0 & 0 & -1\end{array}\right)$

and choose a diagonal tetrad basis $e_{\mu}^{(a)}$,

$e_{\mu}^{(a)}=\left(\begin{array}{cccc}\Lambda(r) & 0 & 0 & 0 \\ 0 & \Lambda(r) & 0 & 0 \\ 0 & 0 & r \Lambda(r)^{-1} & 0 \\ 0 & 0 & 0 & \Lambda(r)\end{array}\right)$,

for which Eq. (1) is satisfied, and then it is easy to determine its inverse form, $e_{(a)}^{\mu}$,

$e_{(a)}^{\mu}=\left(\begin{array}{cccc}\Lambda(r)^{-1} & 0 & 0 & 0 \\ 0 & \Lambda(r)^{-1} & 0 & 0 \\ 0 & 0 & \Lambda(r) / r & 0 \\ 0 & 0 & 0 & \Lambda(r)^{-1}\end{array}\right)$

We are interested in studying the effect of a magnetic field $B_{0}$ that modifies the space-time geometry, in the Melvin metric. If we consider a Dirac particle inside this field, we may also investigate the effect of the minimal coupling in the wave equation for this particle considering the 4-potential $A_{\mu}$. In a flat space-time, a constant magnetic field in the $z$ direction, $B_{0}$, which may be related to an equivalent magnetic field in the Melvin metric by Eq. (2) appears if the 4-potential has only the non-vanishing component

$A_{\phi}=-\frac{2}{B_{0} \Lambda(r)}$.

Observing that the term $\gamma^{\mu} \Omega_{\mu}$, in the curved space wave equation, relative to the tetrad (19) is given by

$\gamma^{\mu} \Omega_{\mu}=\frac{\gamma^{(1)}}{2 \Lambda^{2}} \frac{\partial \Lambda}{\partial r}+\frac{\gamma^{(1)}}{2 r \Lambda}$,
Eq. (13) becomes

$$
\begin{aligned}
& {\left[\frac{\gamma^{(0)}}{\Lambda} \frac{\partial}{\partial t}+\frac{\gamma^{(1)}}{\Lambda}\left(\frac{\partial}{\partial r}+\frac{1}{2 \Lambda} \frac{\partial \Lambda}{\partial r}+\frac{1}{2 r}\right)\right.} \\
& \left.+\frac{\gamma^{(2)} \Lambda}{r} \frac{\partial}{\partial \phi}+\frac{\gamma^{(3)}}{\Lambda} \frac{\partial}{\partial z}+i M+i q \frac{\gamma^{(2)} \Lambda A_{\phi}}{r}\right] \psi=0 .
\end{aligned}
$$

In the limit $\Lambda \rightarrow 1$, this equation reduces to the usual Dirac equation for a free particle in a flat space-time in a cylindrical coordinate system. So, we may interpret Eq. (23) as a generalization of a Dirac equation for a particle inside a magnetic field, an equation that has been extensively studied in the literature, in many contexts, as for example [25-29]. We are interested in observing the corrections in the energy spectrum, due to the alteration of the geometry of the spacetime, determined in Eq. (23).

Making a transformation in Eq. (23),

$\psi=\frac{1}{\sqrt{r \Lambda(r)}} \Phi$

we obtain a simplified form,

$$
\begin{aligned}
& {\left[\frac{\gamma^{(0)}}{\Lambda} \frac{\partial}{\partial t}+\frac{\gamma^{(1)}}{\Lambda} \frac{\partial}{\partial r}+\frac{\gamma^{(2)} \Lambda}{r} \frac{\partial}{\partial \phi}\right.} \\
& \left.+\frac{\gamma^{(3)}}{\Lambda} \frac{\partial}{\partial z}+i M+i q \frac{\gamma^{(2)} \Lambda A_{\phi}}{r}\right] \psi=0,
\end{aligned}
$$

where the usual representation for the gamma matrices is considered:

$\begin{array}{ll}\gamma^{(0)}=\left(\begin{array}{cccc}1 & 0 & 0 & 0 \\ 0 & 1 & 0 & 0 \\ 0 & 0 & -1 & 0 \\ 0 & 0 & 0 & -1\end{array}\right), & \gamma^{(1)}=\left(\begin{array}{cccc}0 & 0 & 0 & 1 \\ 0 & 0 & 1 & 0 \\ 0 & -1 & 0 & 0 \\ -1 & 0 & 0 & 0\end{array}\right), \\ \gamma^{(2)}=\left(\begin{array}{cccc}0 & 0 & 0 & -i \\ 0 & 0 & i & 0 \\ 0 & i & 0 & 0 \\ -i & 0 & 0 & 0\end{array}\right), \quad \gamma^{(3)}=\left(\begin{array}{cccc}0 & 0 & 1 & 0 \\ 0 & 0 & 0 & -1 \\ -1 & 0 & 0 & 0 \\ 0 & 1 & 0 & 0\end{array}\right),\end{array}$

which has no dependence on the $z, t$, and $\phi$ variables. We will assume a solution of the form

$\Phi=R(r) \exp \left[-i \sigma t+i p_{z} z+i m \phi\right]$,

where $\sigma$ is the energy of the system, which assumes positive values for particles and negative values for antiparticles, $p_{z}$ is the momentum, $m= \pm 1, \pm 2, \pm 3, \ldots$, a quantum number, and $M$ is the electron mass. So, Eq. (23) may be written in explicit form: 


$$
\begin{aligned}
& i\left(+\frac{d}{\mathrm{~d} r}+\frac{m}{r} \Lambda^{2}-\frac{q}{r} A_{\phi} \Lambda^{2}\right) \\
& \quad \times R_{4}+(+\sigma-M \Lambda) R_{1}-p_{z} R_{3}=0, \\
& i\left(+\frac{d}{\mathrm{~d} r}-\frac{m}{r} \Lambda^{2}+\frac{q}{r} A_{\phi} \Lambda^{2}\right) \\
& \quad \times R_{3}+(+\sigma-M \Lambda) R_{2}+p_{z} R_{4}=0, \\
& i\left(-\frac{d}{\mathrm{~d} r}-\frac{m}{r} \Lambda^{2}+\frac{q}{r} A_{\phi} \Lambda^{2}\right) \\
& \quad \times R_{2}+(-\sigma-M \Lambda) R_{3}+p_{z} R_{1}=0, \\
& i\left(-\frac{d}{\mathrm{~d} r}+\frac{m}{r} \Lambda^{2}-\frac{q}{r} A_{\phi} \Lambda^{2}\right) \\
& \quad \times R_{1}+(-\sigma-M \Lambda) R_{4}-p_{z} R_{2}=0 .
\end{aligned}
$$

In a first approximation we will solve the equation neglecting terms in higher orders of $r$, and then the system of equations may be written as

$$
\begin{aligned}
- & i\left(+\frac{d}{\mathrm{~d} r}+\frac{m}{r}+\frac{m B_{0}^{2}}{2} r-\frac{2 q}{B_{0} r}-\frac{q B_{0}}{2} r\right) \\
& \times R_{4}+(-\sigma+M) R_{1}+p_{z} R_{3}=0, \\
- & i\left(+\frac{d}{\mathrm{~d} r}-\frac{m}{r}-\frac{m B_{0}^{2}}{2} r+\frac{2 q}{B_{0} r}+\frac{q B_{0}}{2} r\right) \\
& \times R_{3}+(-\sigma+M) R_{2}-p_{z} R_{4}=0, \\
- & i\left(+\frac{d}{\mathrm{~d} r}+\frac{m}{r}+\frac{m B_{0}^{2}}{2} r-\frac{2 q}{B_{0} r}-\frac{q B_{0}}{2} r\right) \\
& \times R_{2}+(-\sigma-M) R_{3}+p_{z} R_{1}=0, \\
- & i\left(+\frac{d}{\mathrm{~d} r}-\frac{m}{r}-\frac{m B_{0}^{2}}{2} r+\frac{2 q}{B_{0} r}+\frac{q B_{0}}{2} r\right) \\
& \times R_{1}+(-\sigma-M) R_{4}-p_{z} R_{2}=0 .
\end{aligned}
$$

This set of equations may be decoupled by multiplying the first equation by the expression

$$
-i\left(+\frac{d}{\mathrm{~d} r}-\frac{m}{r}-\frac{m B_{0}^{2}}{2} r+\frac{2 q}{B_{0} r}+\frac{q B_{0}}{2} r\right)
$$

and then using the second and the fourth to eliminate the terms containing the spinors $R_{1}, R_{2}$, and $R_{3}$. The result is

$$
\begin{aligned}
& {\left[\frac{d^{2}}{\mathrm{~d} r^{2}}-\frac{m^{\prime}\left(m^{\prime}+1\right)}{r^{2}}+b\left(-m^{\prime} \pm \frac{1}{2}\right)\right.} \\
& \left.\quad-\frac{b^{2} r^{2}}{4}+\left(\sigma^{2}-M^{2}-p_{z}^{2}\right)\right] R_{4}(r)=0
\end{aligned}
$$

where $b=m B_{0}^{2}-q B_{0}$ and $m^{\prime}=m-2 q / B_{0}$. In a similar way we derive equations for $R_{1}, R_{2}, R_{3}$ that may be resumed in the form

$$
\begin{aligned}
& {\left[\frac{d^{2}}{\mathrm{~d} r^{2}}-\frac{m^{\prime}\left(m^{\prime} \pm 1\right)}{r^{2}}+b\left(-m^{\prime}+\frac{1}{2}\right)\right.} \\
& \left.-\frac{b^{2} r^{2}}{4}+\left(\sigma^{2}-M^{2}-p_{z}^{2}\right)\right] R(r)=0,
\end{aligned}
$$

where the positive sign refers to $R_{2}(r)$ and $R_{4}(r)$, and the negative one to $R_{1}(r)$ and $R_{3}(r)$.

As we may observe, Eq. (38) is similar to the Schrödinger equation, and it may be written as

$\frac{d^{2} R(r)}{\mathrm{d} r^{2}}+\left(E-V_{\mathrm{ef}}\right) R(r)=0$,

where $E=\sigma^{2}-M^{2}-p_{z}^{2}$. The term $V_{\text {ef }}=\frac{m^{\prime}\left(m^{\prime} \pm 1\right)}{r^{2}}-$ $b\left(-m^{\prime} \pm \frac{1}{2}\right)+\frac{b^{2} r^{2}}{4}$ may be identified as an effective potential and as we can see, the system has the form of an isotropic harmonic oscillator.

In fact, the solution of Eq. (39) may be mapped into a 3dimensional harmonic oscillator-like one in spherical coordinates. These solutions are given in terms of the associated Laguerre polynomials,

$R(S)=\left(\begin{array}{c}N_{1} S^{\left(1 / 2-m^{\prime} / 2\right)} e^{-\frac{S}{2}} L_{n}^{1 / 2-m^{\prime}} \\ N_{2} S^{-m^{\prime} / 2} e^{-\frac{S}{2}} L_{n}^{-1 / 2-m^{\prime}} \\ N_{3} S^{\left(1 / 2-m^{\prime} / 2\right)} e^{-\frac{S}{2}} L_{n}^{1 / 2-m^{\prime}} \\ N_{4} S^{-m^{\prime} / 2} e^{-\frac{S}{2}} L_{n}^{-1 / 2-m^{\prime}}\end{array}\right)$,

where $S=\frac{b r^{2}}{2}$ and $N_{i}$ are normalization constants. The energy spectrum relating to this solution is

$E=\left(n+\frac{3}{2}\right) \frac{b}{2}, \quad n=0,1,2,3, \ldots$,

and using the definition of $E$ given in Eq. (39), we obtain the energy spectrum,

$\sigma=\sqrt{M^{2}+p_{z}^{2}+2 B_{0} q\left(n+s+\frac{1}{2}\right)+2 B_{0}^{2} m\left(n+s+\frac{1}{2}\right)}$,

where $s= \pm 1 / 2$ is the spin quantum number of the particle and $m=1,2,3 \ldots$, in order to keep the conservation of the parity. As was pointed out before, Eq. (41) is written with $c=G=\hbar=1$. By making the conversion to the international system of units we have 
Table 1 Energy levels in the Melvin metric with a minimal coupling, from the value of the magnetic field found in the Earth, up to the one expected to be produced in ultra-relativistic heavy-ion collisions

\begin{tabular}{llll}
\hline & $E_{3}(\mathrm{TeV})$ & $E_{1000}(\mathrm{TeV})$ & $E_{10}^{30}(\mathrm{TeV})$ \\
\hline$B_{\text {Earth }} \sim 0.56 \mathrm{G}$ & $5.109990646783791 \times 10^{-7}$ & $5.109991223884968 \times 10^{-7}$ & 7691.365748802196 \\
$B_{\text {Sum }} \sim 1.5 \mathrm{G}$ & $5.109990650256817 \times 10^{-7}$ & $5.109992381560156 \times 10^{-7}$ & 13321.83625652045 \\
$B_{\text {Pulsar }} \sim 10^{13} \mathrm{G}$ & 0.00005957925431687425 & 0.001087723495543327 & $3.439683331294733 \times 10^{10}$ \\
$B_{\text {Magnetar }} \sim 10^{15} \mathrm{G}$ & 0.0005957708482387581 & 0.01087723376713101 & $3.43968337759476 \times 10^{11}$ \\
$B_{\text {Ions }} \sim 10^{19} \mathrm{G}$ & 0.05957706291159353 & 1.087723375512916 & $3.440150976087315 \times 10^{13}$ \\
\hline
\end{tabular}

Table 2 Energy levels in a flat space-time

\begin{tabular}{llll}
\hline & $E_{3}(\mathrm{TeV})$ & $E_{1000}(\mathrm{TeV})$ & $E_{10}^{30}(\mathrm{TeV})$ \\
\hline$B_{\text {Earth }} \sim 0.56 \mathrm{G}$ & $5.109990646783791 \times 10^{-7}$ & $5.109991223884968 \times 10^{-7}$ & 7691.365748802196 \\
$B_{\text {Sum }} \sim 1.5 \mathrm{G}$ & $5.109990650256817 \times 10^{-7}$ & $5.109992381560156 \times 10^{-7}$ & 13321.83625652045 \\
$B_{\text {Pulsar }} \sim 10^{13} \mathrm{G}$ & 0.00005957925431687425 & 0.001087723495543327 & $3.439683330827056 \times 10^{10}$ \\
$B_{\text {Magnetar }} \sim 10^{15} \mathrm{G}$ & 0.0005957708482387581 & 0.01087723376713101 & $3.439683330827056 \times 10^{11}$ \\
$B_{\text {Ions }} \sim 10^{19} \mathrm{G}$ & 0.05957706291159353 & 1.087723375512916 & $3.439683330827056 \times 10^{13}$ \\
\hline
\end{tabular}

$\sigma=\sqrt{M^{2} c^{4}+p_{z}^{2} c^{2}+2 \hbar c^{2} B_{0} q\left(n+s+\frac{1}{2}\right)+4 \pi \varepsilon_{0} \hbar^{2} G B_{0}^{2} m\left(n+s+\frac{1}{2}\right)}$,

where $\varepsilon_{0}$ is the vacuum permissivity constant. In a system of units with $\hbar=c=1$ we have

$\sigma=\sqrt{M^{2}+p_{z}^{2}+2 B_{0} q\left(n+s+\frac{1}{2}\right)+G B_{0}^{2} m\left(n+s+\frac{1}{2}\right)}$,

where, in the last term inside the square root, the Planck mass $m_{p}$ appears scaling the magnetic field as $\left(B_{0} / m_{p}\right)^{2}$.

\section{Results}

Studying Eq. (41) we may recover some results from the literature. When the magnetic field and $p_{z}$ goes to zero, we obtain the expression for the rest energy of the particle,

$\sigma=M c^{2}$

Now, if the gravitational energy is neglected, the last term inside the square root vanishes and we have

$\sigma=\sqrt{M^{2} c^{4}+p_{z}^{2} c^{2}+2 \hbar c^{2} B_{0} q\left(n+s+\frac{1}{2}\right)}$,

which corresponds to the usual spectrum obtained from a Dirac equation in a flat space with a vector potential. This fact confirms our initial considerations about Eq. (23), interpreting it as a generalization of this equation. It is also possible to analyze the situation where only the gravitational term is considered,

$\sigma=\sqrt{M^{2} c^{4}+p_{z}^{2} c^{2}+4 \pi \varepsilon_{0} \hbar^{2} G B_{0}^{2} m\left(n+s+\frac{1}{2}\right)}$,

and observing this equation it is easy to see that this term (when the magnetic field appears) is a negligible correction for usual magnetic fields (not so strong) for all energy levels.

But we are interested in studying the effect of extreme magnetic fields, as intense as the ones found in magnetars, of the order of $B \sim 10^{15}-10^{16} \mathrm{G}[1,2]$ or the fields expected to be produced in heavy-ion collisions, $B \sim 10^{19} \mathrm{G}[3-5]$. The results for some energy levels, obtained with the exact numerical solution of Eq. (23) (Melvin metric), considering some systems of interest, are shown in Table 1. Observing the table, we can see that the effect of the magnetic field appears when $B$ increases and becomes important when these fields are intense.

The same calculations have been performed in a flat spacetime with a minimal coupling. The results are shown in Table 2. As we can see, the results are essentially the same, and, when comparing them with the ones found in Table 1, we can only find deviations when considering fields of the order of $B \sim 10^{19} \mathrm{G}$ and quantum numbers as large as $n \sim 10^{30}$. These results are shown in Fig. 1. Observing these results, the conclusion that we may obtain, to a very good precision, is that the effect of the magnetic field in the metric is very small and may be neglected. 
Fig. 1 Energy levels as functions of the quantum number $n$ considering Eqs. (41), (45) and Eq. (41) without a minimal coupling (solid line)

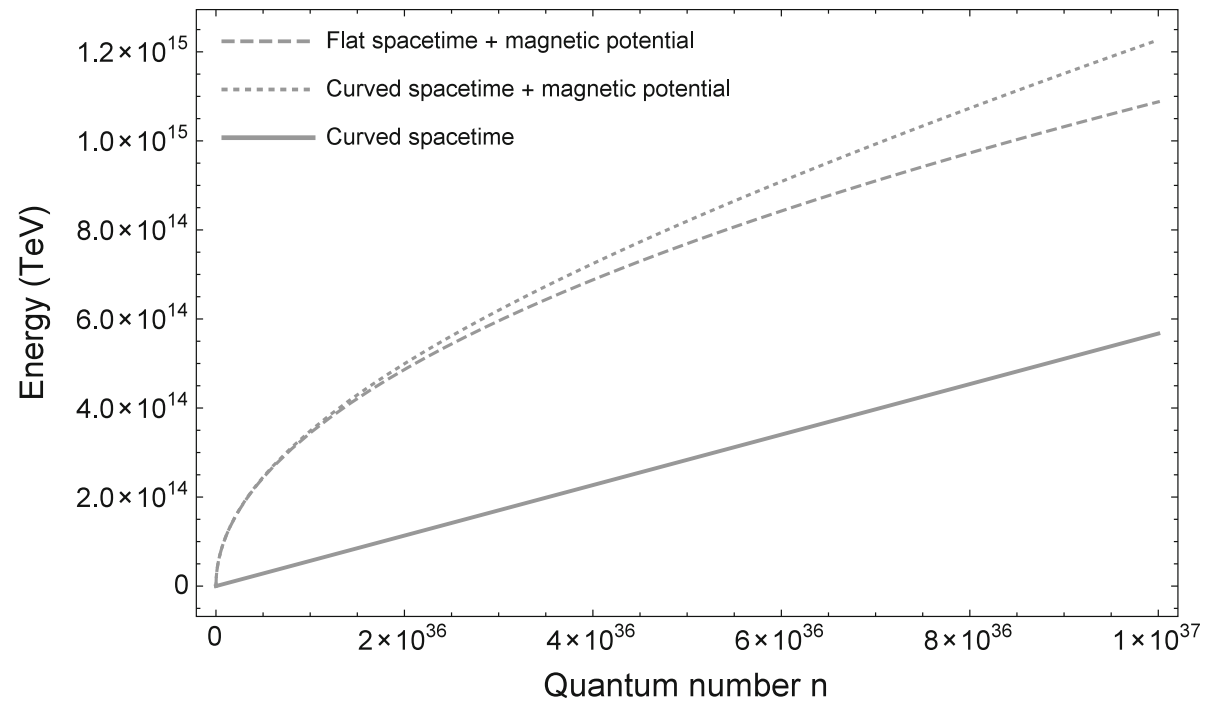

As explained before, Eq. (41) is an approximation for small values of $r$, but when comparing the results with the exact numeric calculations that have been performed, up to the precision shown in Tables 1 and 2 the conclusions that we obtain are essentially the same, and then we may use this equation as a very good approximation. The corrections from terms with higher powers of $r$ appear beyond the precision shown in these tables. This fact may be seen if one observes the approximations that have been made when Eqs. (32)-(35) have been obtained. Terms of the order $\left(B_{0} r\right)^{2 n}$, where $n$ is an integer, appears. These terms increase with $r^{2}$, and they reach the maximum values with some $r_{M}$, which determines the size of the considered system. For heavyion collisions, for example, $\left(B_{0} r_{M}\right)^{2} \sim 10^{-33}$, for pulsars, $10^{-11}$. The greater value obtained was for magnetars, $10^{-7}$, and the effect of these corrections, as said before, are always beyond the precision shown in the tables, so, we may conclude that the accuracy of the approximation is good, even for extremely large magnetic fields and levels.

One remark that must be made is that when considering a covariant Dirac equation, a particle with gyromagnetic ratio $g=2$ is taken into account. This is a good approximation for electrons inside stars, for example. But when considering higher energy processes, as high-energy collisions, deviations from this value are proposed to exist, and then one way to study this question is to consider extensions of the covariant Dirac equation, as for example has been made in [21]. This kind of approach is left for future work.

Another aspect that may be taken into account is the Melvin metric. It is clear that many of the systems that have been studied do not have a magnetic field of the form of the one that determines this metric, but in some regions, with intense magnetic fields, at least as a first qualitative approximation, these results must be correct, and we expect that more careful calculations, with the metric determined by different shapes of the magnetic fields, confirm our results.

Open Access This article is distributed under the terms of the Creative Commons Attribution 4.0 International License (http://creativecomm ons.org/licenses/by/4.0/), which permits unrestricted use, distribution, and reproduction in any medium, provided you give appropriate credit to the original author(s) and the source, provide a link to the Creative Commons license, and indicate if changes were made.

Funded by $\mathrm{SCOAP}^{3}$.

\section{References}

1. C. Thompson, R.C. Duncan, Mon. Not. R. Astron. Soc. 275, 255 (1995)

2. R.F. Archibald et al., Nature 497, 591 (2013)

3. A. Bzdak, V.V. Skokov, Phys. Lett. B 710, 171 (2012)

4. V. Voronyuk, V.D. Toneev, W. Cassing, E.L. Bratkovskaya, V.P. Konchakovsky, S.A. Voloshin, Phys. Rev. C 83, 054911 (2011)

5. U. Gursoy, D. Kharzeev, K. Rajagopal, Phys. Rev. C 89, 054905 (2014)

6. S.S. Avancini, C.C. Barros Jr., D.P. Menezes, C. Providência, Phys. Rev. C 82, 025808 (2010)

7. D.P. Menezes, C. Providência, M. Chiapparini, M.E. Bracco, A. Delfino, M. Malheiro, Phys. Rev. C 76, 064902 (2007)

8. M.A. Melvin, Phys. Lett. 8, 65 (1964)

9. M.A. Melvin, Phys. Rev. B 139, 225 (1965)

10. W.B. Bonnor, Proc. Phys. Soc. A 67, 225 (1954)

11. K.S. Thorne, Phys. Rev. B 139, 244 (1965)

12. H. Kadlecova, Phys. Rev. D 82, 044041 (2010)

13. T.I. Gutsunaev, V. Manko, Phys. Lett. A 123, 215 (1987)

14. T.I. Gutsunaev, V. Manko, Phys. Lett. A 132, 85 (1988)

15. Y.N. Obukhov, A.J. Silenko, O.V. Teryaev, Phys. Rev. D 80, 064044 (2009)

16. Y.N. Obukhov, A.J. Silenko, O.V. Teryaev, Phys. Rev. D 88, 084014 (2013)

17. J. Schwinger, Phys. Rev. 130, 800 (1963)

18. M. Nakahara, Geometry, Topology and Physics (Taylor and Francis, Abingdon, 2003) 
19. F.W. Hehl, J. Lemke, E.W. Mielke, Two lectures on fermions and gravity, in Geometry and Theoretical Physics, Proc. of the Bad Honnef School 12-16 Feb. 1990, ed. by J. Debrus, A.C. Hirshfeld (Springer, Heidelberg, 1991), pp. 56-140

20. M. Blagojevic, F.W. Hehl (eds.), Gauge Theories of Gravitation. A Reader with Commentaries (Imperial College Press, London, 2013)

21. Y.N. Obukhov, A.J. Silenko, O.V. Teryaev, Phys. Rev. D 90, 124068 (2014)
22. F.J. Ernst, J. Math. Phys. 17, 54 (1976)

23. M.A. Melvin, Ann. N. Y. Acad. Sci. 262(2), 253 (1975)

24. D. Kastor, J. Traschen, Class. Quantum Grav. 31, 075023 (2014)

25. D. Melrose, A. Parle, Aust. J. Phys. 36, 755 (1983)

26. M.H. Johnson, B.A. Lippmann, Phys. Rev. 76, 828 (1949)

27. B.P. Mandal, S. Verma, Phys. Lett. A 374, 1021 (2010)

28. H.K. Lee, Y. Yoon, J. High Energy Phys. 2007, 86 (2007)

29. S.N. Datta, A. Misra, J. Chem Phys. 114, 1478 (2001) 